\title{
Hypoxia promotes redifferentiation and suppresses markers of hypertrophy and degeneration in both healthy and osteoarthritic chondrocytes
}

Brandon D Markway, Holly Cho and Brian Johnstone*

\begin{abstract}
Introduction: Hypoxia is considered to be a positive influence on the healthy chondrocyte phenotype and cartilage matrix formation. However, hypoxia-inducible factors (HIFs) have been implicated in the pathogenesis of osteoarthritis (OA). Thus, we assessed whether healthy and OA chondrocytes have distinct responses to oxygen, particularly with regard to hypertrophy and degradation during redifferentiation.
\end{abstract}

Methods: Monolayer-expanded healthy and OA chondrocytes were redifferentiated for 14 days in pellet cultures under standard (20\% oxygen) or hypoxic ( $2 \%$ oxygen) conditions. Cartilage matrix gene expression, matrix quality and quantity, degradative enzyme expression and HIF expression were measured.

Results: In hypoxia, both healthy and OA chondrocytes had higher human collagen type II, $\alpha 1$ gene (COL2A1), and aggrecan (ACAN) expression and sulfated glycosaminoglycan (sGAG) accumulation, concomitant with lower human collagen type $X, \alpha 1$ gene (COL1OA1), and human collagen type I, $\alpha 1$ gene (COL1A1), expression and collagen I extracellular accumulation. OA chondrocytes had significantly lower sGAGs/DNA than healthy chondrocytes, but only in high oxygen conditions. Hypoxia also caused significantly greater sGAG retention and hyaluronic acid synthase 2 (HAS2) expression by OA chondrocytes. Both healthy and OA chondrocytes had significantly lower expression of matrix metalloproteinases (MMPs) MMP1, MMP2, MMP3 and MMP13 in hypoxia and less active MMP2 enzyme, consistent with lower MMP14 expression. However, aggrecanase (ADAMTS4 and ADAMTS5) expression was significantly lowered by hypoxia only in healthy cells, and COL1OA1 and MMP13 remained significantly higher in OA chondrocytes than in healthy chondrocytes in hypoxic conditions. HIF- $1 \alpha$ and HIF- $2 \alpha$ had similar expression profiles in healthy and OA cells, increasing to maximal levels early in hypoxia and decreasing over time.

Conclusions: Hypoxic culture of human chondrocytes has long been acknowledged to result in increased matrix accumulation, but still little is known of its effects on catabolism. We show herein that the increased expression of matrix proteins, combined with decreased expression of numerous degradative enzymes by hypoxia, minimizes but does not abolish differences between redifferentiated healthy and OA chondrocytes. Hypoxia-induced HIF expression is associated with hypertrophic marker and degradative enzyme downregulation and increased measures of redifferentiation in both healthy and OA chondrocytes. Therefore, though HIFs may be involved in the pathogenesis of OA, conditions that promote HIF expression in vitro promote matrix accumulation and decrease degradation and hypertrophy, even in cells from OA joints.

\footnotetext{
* Correspondence: johnstob@ohsu.edu

Department of Orthopaedics \& Rehabilitation, Oregon Health \& Science

University, 3181 SW Sam Jackson Park Road, OP31, Portland, OR 97239 USA
} 


\section{Introduction}

The proper application of cellular therapies for articular cartilage repair is hindered by a lack of clarity with regard to the mechanisms that underlie the development and maintenance of the permanent chondrocyte phenotype as opposed to the transient endochondral phenotype. During endochondral ossification in development, transient chondrocytes of the anlagen undergo hypertrophy prior to apoptosis and replacement by bone. It is argued that hypertrophy is also a feature of osteoarthritis (OA) because the permanent chondrocytes of articular cartilage can express markers of hypertrophic endochondral chondrocytes, such as collagen X (COL1OA1) and matrix metalloproteinase 13 (MMP13) during degeneration. The use of chondrocytes for cartilage repair requires in vitro expansion, a process that leads to dedifferentiation whereby the chondrocytes decrease expression of cartilage matrix genes such as aggrecan $(A C A N)$ and collagen II (COL2A1), with collagen I (COL1A1) becoming the predominant collagen type [1]. Although it has long been established that these chondrocytes can be redifferentiated in three-dimensional culture [2], this may not be completely successful, because hypertrophy-related markers can also be upregulated, even in chondrocytes from nondiseased joints [3,4]. Therefore, achieving a better understanding of the factors regulating hypertrophy has important implications both for tissue engineering and for treatment of OA.

Although it is known that oxygen levels and the PerArnt-Sim (PAS) family of transcription factors known as hypoxia-inducible factors (HIFs), particularly HIF- $1 \alpha$ and HIF- $2 \alpha$, play an active role in chondrocyte biology, their precise contributions to both cartilage maintenance and the progression of disease remain unclear [5-7]. These $\alpha$-subunits are subject to degradation in the presence of sufficient oxygen, but in hypoxic environments such as the avascular joint, their stabilization allows heterodimerization and transactivation of hypoxia-responsive target genes. In vitro hypoxic culture of healthy human chondrocytes or cartilage explants causes an increase in HIF expression and promotes chondrogenic matrix genes [8-10] while suppressing MMP-1 and MMP-13 expression and activity [10,11] and decreasing ADAMTS5 mRNA expression and aggrecanase-mediated degeneration [10]. However, recent data suggest that HIF- $2 \alpha$ can promote expression of genes involved in cartilage degeneration and hypertrophy $[12,13]$. The potential relevance of oxygen-dependent signaling to OA has been acknowledged for some time. In the past decade, multiple groups have reported elevated HIF- $1 \alpha$ expression in degenerated cartilage [14-16]. However, two groups have independently identified numerous hypertrophic and OA-associated genes as targets of HIF- $2 \alpha$ regulation, including COL10A1 and matrix metalloproteinases MMP1, MMP3, MMP9 and MMP13, using reporter gene assays [12,13]. These studies provoked considerable speculation about the importance of HIF- $2 \alpha$ in hypertrophy and OA [17], but other studies have raised questions about its role in these processes [5,7]. For example, Araldi et al. found that conditional knockout of Epas1 (the HIF-2 $\alpha$ gene) had minimal effects on Col10a1 expression [5]. As noted in a commentary on the initial HIF- $2 \alpha /$ hypertrophy reports, the data are from in vitro overexpression studies conducted in $20 \%$ oxygen [6]. Because oxygen-dependent posttranslational regulation of HIFs is thought to be the primary means of control, such culture conditions may have influenced the observed downstream effects.

In our present study, we investigated the effects of oxygen on three-dimensional redifferentiation in both healthy and OA chondrocytes. The proteomes of dedifferentiated OA and healthy chondrocytes were reported to be differentially modulated by changes in oxygen tension using cells in monolayer culture [18] and more recently were shown to differentially respond to hypoxia when cultured with interleukin $1 \beta$ (IL-1 $\beta$ ) [19]; however, we are aware of no such comparison during three-dimensional redifferentiation published to date. Three-dimensional culture of chondrocytes in defined medium promotes matrix accumulation and redifferentiation [20]. Although this is considered preferable for chondrocytes that are to be implanted, it can also increase expression of hypertrophic genes. For example, Dehne et al. found that runt-related transcription factor 2 (RUNX2) and COL1OA1 were both increased during redifferentiation, regardless of whether the cells were of nondiseased or osteoarthritic origin [3]. An increase in COL10A1 upon three-dimensional culture was also reported for chondrocytes from both total joint replacement knees and autologous chondrocyte implantation surplus cartilage [4]. Because three-dimensional culture can promote redifferentiation while also increasing markers of hypertrophy, this system allows a comparative analysis of the hypertrophic and degradative response that could be missed in monolayer culture. We hypothesized that this response might be differentially regulated by hypoxia in OA and healthy chondrocytes concomitant with different HIF expression patterns. To investigate this possibility, we evaluated healthy and OA chondrocytes' hypertrophic and degradative responses to hypoxia ( $2 \%$ oxygen), as well as the nature and quantity of matrix production and expression of HIFs.

\section{Materials and methods}

\section{Isolation and expansion of human chondrocytes}

Healthy chondrocytes were harvested from normal cadaver femoral condyles and OA chondrocytes from tissue taken during total joint replacement surgery $(n=5$ each; for representative images, see Additional file 1). Human tissue was obtained as discard tissue without 
patient identifiers. The Oregon Health \& Science University Institutional Review Board approved the study as being exempt from the requirement for consent. Cartilage was scraped from the condyles and finely minced before enzymatic digestion. Cartilage digestion was initiated with $1 \%$ protease (wt/vol) from Streptomyces griseus in low-glucose Dulbecco's modified Eagle's medium (DMEM; Life Technologies, Grand Island, NY, USA) supplemented with $1 \%$ penicillin-streptomycin $(\mathrm{P} / \mathrm{S})$. After $1 \mathrm{~h}$ at $37^{\circ} \mathrm{C}$, protease was removed and replaced with $1,300 \mathrm{U} / \mathrm{ml}$ collagenase II (Worthington Biochemical, Lakewood, NJ, USA) in DMEM + P/S for $3 \mathrm{~h}$ at $37^{\circ} \mathrm{C}$. The cell suspension was passed through a $40-\mu \mathrm{m}$ cell strainer and centrifuged at $500 \times g$ for $5 \mathrm{~min}$, then the cells were resuspended in low-glucose DMEM supplemented with $10 \%$ fetal bovine serum and $1 \% \mathrm{P} / \mathrm{S}$. Chondrocytes were plated at $7,000 / \mathrm{cm}^{2}$ and expanded in monolayer culture in a standard tissue culture incubator with atmospheric oxygen and $5 \% \mathrm{CO}_{2}$.

The storage conditions for osteochondral allografts may affect chondrocyte viability and metabolic activity, although the time at which these effects become evident depends on the storage solution, and in some medium solutions no change is observed even after 2 wk [21]. We obtained OA specimens just hours after total joint replacement, and healthy cartilage was collected from discarded osteochondral allografts of proprietary storage conditions. For the chondrocytes used here, however, we found no significant difference in either the number of viable cells obtained per gram of tissue digested $(5.1 \times$ $10^{6} \pm 3.3 \times 10^{6}$ versus $6.2 \times 10^{6} \pm 3.1 \times 10^{6}$, for healthy and OA cells, respectively) or in the doubling time from initial plating to first passage $(6.9 \pm 2.1$ versus $6.4 \pm 1.5$ for healthy and OA cells, respectively).

\section{Pellet culture redifferentiation of chondrocytes}

Chondrocytes were redifferentiated between the first and third passages with serum-free chondrogenic induction medium consisting of high-glucose DMEM (Life Technologies) containing $10 \mathrm{ng} / \mathrm{ml}$ transforming growth factor $\beta 1$ (Peprotech, Rocky Hill, NJ, USA), $10^{-7} \mathrm{M}$ dexamethasone, $37.5 \mu \mathrm{g} / \mathrm{ml}$ ascorbic acid 2-phosphate, $1 \mathrm{mM}$ sodium pyruvate, $40 \mu \mathrm{g} / \mathrm{ml}$ L-proline, $1 \times$ ITS + Universal Culture Supplement Premix (BD Biosciences, San Jose, CA, USA) and $1 \% \mathrm{P} / \mathrm{S}$. Pellet cultures were formed by centrifuging $1 \times$ $10^{5}$ cells at $500 \times g$ in $250 \mu \mathrm{l}$ of medium in Nunc polypropylene V-bottom 96-well plates (Thermo Fisher Scientific, Waltham, MA, USA) and maintained in a hypoxic chamber (BioSpherix, Lacona, NY, USA) set at $2 \%$ oxygen, $5 \%$ $\mathrm{CO}_{2}$ or a standard tissue culture incubator with $5 \% \mathrm{CO}_{2}$. Although $\mathrm{CO}_{2}$ displacement actually lowers the oxygen level, hereafter we refer to the standard condition as $20 \%$ oxygen according to convention. Medium was changed every 2 or 3 days, with that of the hypoxic cultures being done at $2 \%$ oxygen.

\section{Biochemical assays}

All biochemical assays were conducted on at least triplicate pellets. Pellets were rinsed with phosphate-buffered saline (PBS) and digested overnight at $60^{\circ} \mathrm{C}$ in $4 \mathrm{U} / \mathrm{ml}$ papain (Sigma-Aldrich, St Louis, MO, USA) in PBS containing $6 \mathrm{mM} \mathrm{Na}_{2}$-ethylenediaminetetraacetic acid and $6 \mathrm{mM} \mathrm{L-cysteine} \mathrm{(papain} \mathrm{buffer,} \mathrm{pH}$ 6.0). For samples to be assayed for hydroxyproline, iodoacetic acid was added to a final concentration of $10 \mathrm{mM}$ after papain digestion.

DNA content of all papain-digested pellets was quantified using $2 \mu \mathrm{g} / \mathrm{ml}$ Hoechst dye with calf thymus DNA diluted in papain buffer used to prepare standard curves. Briefly, $50 \mu \mathrm{l}$ of samples, standards and blanks were added to black 96-well plates with $200 \mu \mathrm{l}$ of Hoechst dye before fluorescence emission was measured with a multiwell plate reader (excitation $355 \mathrm{~nm}$, emission $455 \mathrm{~nm}$ ).

The sulfated glycosaminoglycan (sGAG) content of pellets and culture medium was quantified using 1,9dimethymethylene blue (DMMB) dye. Supernatant collected at each medium change was used to quantify the total amount of sGAGs produced and lost into the medium. Shark chondroitin sulfate diluted in either DMEM or papain buffer was used to prepare standard curves. For microplate assays, $50 \mu \mathrm{l}$ of samples, standards and blanks were added to clear 96-well plates with $200 \mu \mathrm{l}$ of DMMB dye $(18 \mu \mathrm{g} / \mathrm{ml}$ in $0.5 \%$ ethanol, $0.2 \%$ formic acid, $30 \mathrm{mM}$ sodium formate; $\mathrm{pH}$ 3.0) before absorbance was measured $(575 \mathrm{~nm})$.

Hydroxyproline content of pellets was quantified using an adaptation of the chloramine- $\mathrm{T}$ hydrate oxidation/pdimethylaminobenzaldehyde development method with solid-phase hydrolysis on Dowex 50WX8-400 ion exchange resin (Thermo Fisher Scientific) [22]. Trans-4hydroxy-L-proline in papain buffer was used to generate a standard curve. Absorbance of samples was measured at $560 \mathrm{~nm}$ in a multiwell plate reader.

\section{RNA isolation and real-time quantitative polymerase chain reaction}

The RNeasy Mini Kit (QIAGEN, Germantown, MD, USA) was used to collect total RNA from four to six pellets of each condition. Pooled pellets were snap-frozen in liquid nitrogen and crushed, then immediately lysed with Buffer RLT lysis buffer (QIAGEN) containing $40 \mathrm{mM}$ dithiothreitol (DTT). RNA isolation then proceeded as per the manufacturer's instructions.

RNA samples (250 ng) were reverse-transcribed using qScript cDNA SuperMix (Quanta BioSciences, Gaithersburg, MD, USA) as per the manufacturer's instructions. 
Quantitative polymerase chain reaction (qPCR) analysis was performed using a MyiQ iCycler thermal cycler (Bio-Rad Laboratories, Hercules, CA, USA) with PerfeCTa qPCR FastMix (Quanta BioSciences) and TaqMan assays (Life Technologies) for ACAN (Hs00153936_m1), COL2A1 (Hs00264051_m1), COL1A1 (Hs00164004_m1), COL10A1 (Hs00166657_m1), MMP1 (Hs00899658_m1), MMP2 (Hs00234422_m1), MMP3 (Hs00968305_m1), MMP9 (Hs00234579_m1), MMP13 (Hs00233992_m1), MMP14 (Hs00237119_m1), TIMP2 (Hs00234278_m1), ADAMTS4 (Hs00192708_m1), ADAMTS5 (Hs00199841_m1), HAS2 (Hs00193435_m1), HIF1A (Hs00153153_m1) and EPAS1 (Hs01026149_m1). The cycling parameters were $45^{\circ} \mathrm{C}$ for $2 \mathrm{~min}, 95^{\circ} \mathrm{C}$ for $1 \mathrm{~min}$, then $95^{\circ} \mathrm{C}$ for $5 \mathrm{~s}$ and $60^{\circ} \mathrm{C}$ for $30 \mathrm{~s}$ for a total of 40 cycles. Results were analyzed using the $2^{-\Delta \mathrm{Ct}}$ method relative to the housekeeping gene $18 \mathrm{~S}$ (Hs99999901_s1).

\section{Gelatin zymography}

Gelatin zymography was performed on 10\% SDS-PAGE gels containing $10 \mathrm{mg} / \mathrm{ml}$ gelatin. Gelatin was dissolved in 1.5 M Tris, $\mathrm{pH} 8.8$, and displaced a corresponding volume of 1.5 M Tris during gel preparation. Medium samples from days 9 to 14 of pellet culture were pooled for each oxygen condition. Pooled samples were prepared in Lammeli sample buffer without reducing agents or boiling, and $20 \mu \mathrm{l}$ of this solution was used for zymography. Samples were run alongside PageRuler Plus marker (Thermo Fisher Scientific), then gels were incubated in renaturing buffer (2.7\% Triton X-100) two times for $30 \mathrm{~min}$ each before equilibrating in developing buffer $(50 \mathrm{mM}$ Tris base, $5 \mathrm{mM} \mathrm{CaCl}_{2}, 40 \mathrm{mM} \mathrm{HCl}, 200 \mathrm{mM} \mathrm{NaCl}, 0.02 \%$ (wt/vol) Brij-35) for $30 \mathrm{~min}$ at room temperature. The developing buffer was then changed, and the gels were incubated at $37^{\circ} \mathrm{C}$ for $24 \mathrm{~h}$. Developed zymograms were stained with Coomassie Blue (0.1\% Coomassie R-250, 10\% acetic acid, $40 \%$ methanol) for $45 \mathrm{~min}$, then destained in a solution of $10 \%$ acetic acid and $20 \%$ methanol until bands were resolved.

\section{Immunohistochemical analysis}

Pellets were fixed in $10 \%$ neutral buffered formalin, embedded in paraffin and sectioned onto slides. Sections were deparaffinized and treated with $1 \mathrm{mg} / \mathrm{ml}$ protease in PBS for $30 \mathrm{~min}$ at room temperature. For collagen I and collagen II double-staining, protease treatment was followed by $1 \mathrm{mg} / \mathrm{ml}$ hyaluronidase in PBS for $30 \mathrm{~min}$ at $37^{\circ} \mathrm{C}$. Slides blocked with $5 \%$ bovine serum albumin (BSA) were probed overnight at $4^{\circ} \mathrm{C}$ with primary antibodies to collagen I (rabbit polyclonal antibody, 1:200; kind gift from A Hollander, University of Bristol, UK), collagen II (II-II6B3 mouse monoclonal antibody, 1:200; Developmental Studies Hybridoma Bank, University of
Iowa, Iowa City, IA, USA), and collagen X (mouse polyclonal antibody, 1:300; kind gift from GJ Gibson, Henry Ford Hospital, Detroit, MI, USA) diluted in 1\% BSA. Secondary antibodies (Oregon Green-conjugated antirabbit (1:250) and Alexa Fluor 596-conjugated antimouse (1:500) for double-staining; fluorescein isothiocyanate-conjugated anti-mouse diluted 1:500 for collagen X) were added in 1\% BSA for $45 \mathrm{~min}$ at room temperature. Slides were mounted with ProLong Gold antifade reagent containing 4',6-diamidino-2-phenylindole (Life Technologies) and imaged using an Olympus BX51 microscope (Olympus America, Melville, NY, USA).

\section{Western blot analysis}

Whole-cell lysates were obtained by pooling 20 pellets from each condition at days 2, 7 and 14. Pellets were rinsed with PBS, snap-frozen in liquid nitrogen and crushed. The powdered sample was immediately resuspended in an enhanced radioimmunoprecipitation assay buffer with 1\% SDS and 100 mM DTT [23]. Hypoxic cultures were maintained in low oxygen until directly before freezing.

Lysates were prepared in Laemmli sample buffer with $100 \mathrm{mM}$ DTT and boiled for $3 \mathrm{~min}$. Samples were run on $12 \%$ SDS-PAGE gels for histone $\mathrm{H} 3$ and on $6 \%$ SDSPAGE gels for HIF blots using PageRuler Plus Prestained Protein Ladder (Molecular Weight Marker) (Thermo Fisher Scientific). Gels were transferred to polyvinylidene difluoride and blocked for $1 \mathrm{~h}$ with $2 \%$ milk-PBST $(1 \times$ PBS, $0.1 \%$ Tween 20) at room temperature. Primary antibody incubations were done in blocking solution overnight at $4^{\circ} \mathrm{C}$. All washes were done three times for $10 \mathrm{~min}$ each in PBST.

The quantity of nuclear protein loaded between samples was normalized to histone $\mathrm{H} 3$. The relative signal of bands from a blot with equal volumes of lysates loaded were determined using an Odyssey imaging system (LI-COR Biosciences, Lincoln, NE, USA), and lysate volumes were adjusted accordingly for HIF protein blots. Anti-histone H3 primary antibody (ab24834; Abcam, Cambridge, MA, USA) was used at a 1:500 dilution, and IRDye $800 \mathrm{CW}$ secondary antibody (catalog no. 926-32210; LI-COR Biosciences) was used at a 1:400 dilution. HIF blots were probed with anti-HIF-1 $\alpha$ (1:250 dilution, catalog no. 610958; BD Biosciences) and antiHIF2 $\alpha$ antibodies (1:500 dilution, sc-46691; Santa Cruz Biotechnology, Santa Cruz, CA, USA). To visualize blots, horseradish peroxidase-conjugated secondary antibody (1:2,500 dilution, catalog no. W4021; Promega, Madison, WI, USA) and Western Lightning Plus-ECL (PerkinElmer, Waltham, MA, USA) were used with a Kodak RP X-OMAT Developer (Eastman Kodak Health Imaging, Rochester, NY, USA). 


\section{Statistical analysis}

SPSS version 19.0 software (SPSS, Chicago, IL, USA) was used to assess statistical significance, which was defined as $P<0.05$. Normality was evaluated using the Shapiro-Wilk test. Two-tailed independent $t$-tests or paired $t$-tests were used for normally distributed data, and Mann-Whitney $U$ or Wilcoxon tests were used for nonparametric data. The unpaired tests were used to compare means for healthy chondrocytes with those of OA chondrocytes at a given oxygen level, and paired tests were used to compare means between oxygen levels within a given disease condition. The correlation coefficient, Pearson's $r$, for the ratios of mRNA expression of hyaluronic acid synthase 2 (HAS2) and sGAG retention at $2 \%$ oxygen over $20 \%$ oxygen was determined using bivariate analysis.

\section{Results}

Oxygen-dependent expression of cartilage matrix genes

When the expression levels of major matrix genes were compared, healthy and OA chondrocytes had similar responses: COL2A1 and $A C A N$ (Figure 1A, B) were significantly higher at $2 \%$ compared with $20 \%$ oxygen, whereas COL1A1 and COL1OA1 (Figures $1 \mathrm{C}$ and 1D) were significantly lower. Furthermore, the COL2A1: COL1A1 ratio was consistently increased by hypoxia compared to normoxia (9- to 87-fold in healthy pellets and 8- to 54-fold in OA pellets; Additional file 2). Because of the higher variability of the increase in healthy cell pellets, this was not significant at the $\alpha=0.05$ level $(P=0.097 ; P=0.047$ for OA). There was no significant difference in the COL2A1: $C O L 1 A 1$ ratio between healthy and OA cells at either oxygen level. No statistically significant differences were detected in expression levels between healthy and OA chondrocytes at day 0 of pellet cultures, and COL1OA1 was undetectable (data not shown); however, redifferentiation led to differential changes in the two groups. COL $2 A 1$ and $A C A N$ levels were similar between healthy and $\mathrm{OA}$ at both oxygen levels, but COL1A1 and COL1OA1 were greater in OA chondrocyte pellets at $20 \%$ oxygen, a difference that was also significant for COL10A1 in $2 \%$ oxygen.

\section{Oxygen-dependent patterns of collagen distribution}

Regardless of the disease state, oxygen-dependent changes in matrix production and organization detected by immunohistochemical staining were similar (Figure 2). Although pellets from both oxygen levels stained positive for collagen II throughout the matrix, collagen I had distinct oxygen-dependent staining patterns. Pellets cultured at $20 \%$ oxygen had the most intense collagen I staining around the periphery of the pellets, where the cells also had a distinctly elongated (fusiform) morphology. All pellets cultured at $2 \%$ oxygen had qualitatively less collagen
I staining in the matrix than $20 \%$ oxygen pellets, and they also lacked the flattened cells at their periphery. Additionally, the positive signal for collagen I in hypoxic pellets appeared to be intracellular. Collagen X was barely detectable in the matrices of both healthy and OA pellets, but, as with collagen I, the strongest staining was at the periphery of the pellets cultured at $20 \%$ oxygen.

\section{Oxygen-dependent matrix production}

DNA content was used to normalize biochemical measures of matrix production. Although there was no difference between healthy and OA chondrocytes in DNA content at either oxygen level, OA pellets had significantly lower DNA when cultured in $20 \%$ oxygen than in $2 \%$ oxygen $(P=0.030)$ (Figure $3 \mathrm{~A})$. In healthy chondrocytes, DNA content was not significantly different between the two oxygen levels.

In both healthy and OA chondrocyte pellets, the mean hydroxyproline/DNA was higher in $20 \%$ oxygen compared with $2 \%$ oxygen (Figure $3 \mathrm{~B}$ ). In OA pellets, the difference in this measure between the two oxygen levels was significant. Despite the fact that, for each of the five healthy donors' chondrocytes, their $20 \%$ oxygen pellets contained higher hydroxyproline/DNA than their matched $2 \%$ oxygen cultures, this difference was not significant $(P=0.075)$. Hydroxyproline content was not significantly different between healthy and OA chondrocytes at either oxygen level.

Both healthy and OA chondrocytes had significantly more sGAG/DNA in pellets at $2 \%$ compared with those at $20 \%$ oxygen (Figure $3 \mathrm{C}$ ). Although there was no significant difference between healthy and OA chondrocytes at $2 \%$ oxygen, OA pellets had significantly less sGAG/DNA than healthy counterparts at $20 \%$ oxygen (Figure 3C). Culture medium sGAG content was quantified, and the degree of proteoglycan retention was assessed, using sGAGs in pellets/total sGAGs produced (Figure 3D). Not only was the percentage of sGAGs retained within $\mathrm{OA}$ pellets significantly lower than that of healthy pellets in $20 \%$ oxygen, but the percentage retention of sGAGs was also found to be a significant measure of the oxygen response of healthy and OA chondrocytes. Although oxygen level did not affect retention of sGAGs in healthy chondrocyte pellets, OA pellets retained significantly less sGAGs at $20 \%$ oxygen.

\section{Oxygen-dependent expression of catabolic and anabolic enzymes}

Expression of MMP1, MMP3 and MMP13 (Figures 4A through $4 \mathrm{C}$ ) were all significantly lower in $2 \%$ oxygen than in $20 \%$ oxygen cultures for both healthy and OA chondrocytes. MMP13 gene expression was both oxygen- and disease state-dependent. At $20 \%$ oxygen, very 


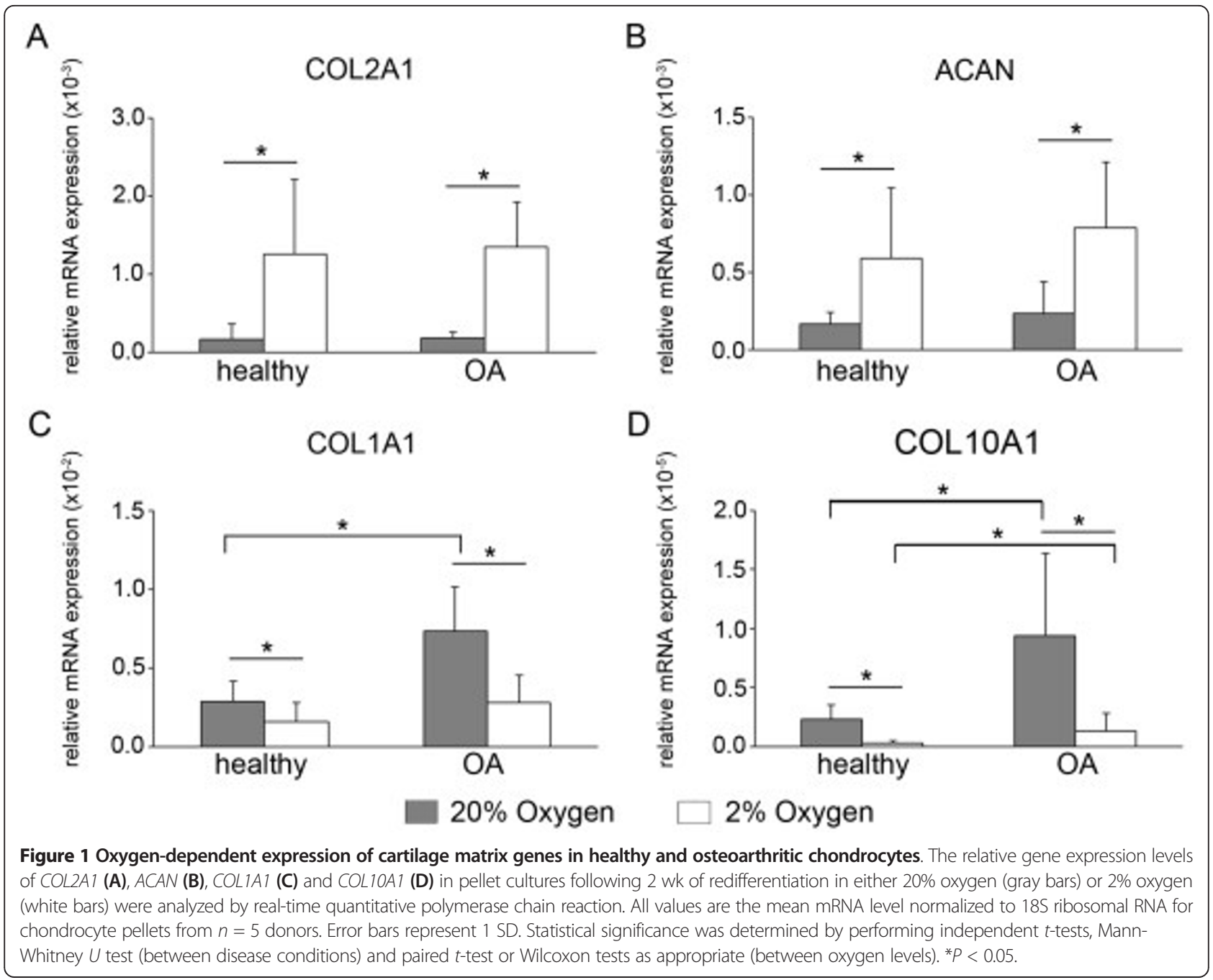

high MMP13 gene expression levels seen in some OA samples resulted in a mean 22 -fold difference between $\mathrm{OA}$ and healthy chondrocytes, but the wide variation meant that statistical significance was not established $(P=0.123)$. At $2 \%$ oxygen, however, $M M P 13$ was significantly higher in OA than in healthy chondrocytes (19-fold). Although these differences were observed in redifferentiated pellets, there was no statistically significant difference in MMP13 expression between healthy and OA cells at day 0 of pellet culture (data not shown).

Like the other MMPs, MMP2 was significantly lower in $2 \%$ oxygen than in $20 \%$ oxygen for both healthy and OA chondrocytes (Figure 4D). Furthermore, gelatin zymography indicated that less of the active form of MMP2 was generated at $2 \%$ oxygen (Figure $4 \mathrm{E}$ ). Additionally, supernatants from healthy cells in $20 \%$ oxygen displayed unidentified gelatinolytic activity near $130 \mathrm{kDa}$. We could not consistently detect MMP9 mRNA expression at either oxygen level in either healthy or OA cells (data not shown), and supernatants did not show gelatinolytic activity in the molecular weight range where MMP9 is typically detected (92 kDa).

Since MMP2 displayed oxygen-dependent generation of its active form, we evaluated expression of the two essential members of the complex responsible for activation of pro-MMP2 at the cell surface, tissue inhibitor of $M M P 2$ (TIMP2) and MMP14 (Figures 4F and 4G). Although TIMP2 was not oxygen-dependent, like the other MMPs evaluated, MMP14 was expressed at a significantly lower level in $2 \%$ oxygen in both healthy and OA cells.

Neither aggrecanase 1 (ADAMTS4) nor aggrecanase 2 (ADAMTS5) (Figures 5A and 5B) had disease state-dependent expression, regardless of the oxygen level. However, expression of both genes showed some degree of oxygendependent regulation. Both ADAMTS4 and ADAMTS5 were significantly higher in healthy chondrocytes at $20 \%$ compared with $2 \%$ oxygen. Whereas their expression was higher at $20 \%$ oxygen for both genes in four of the five OA samples, this difference was not significant $(P=0.119$ and $P=0.127$, respectively). 


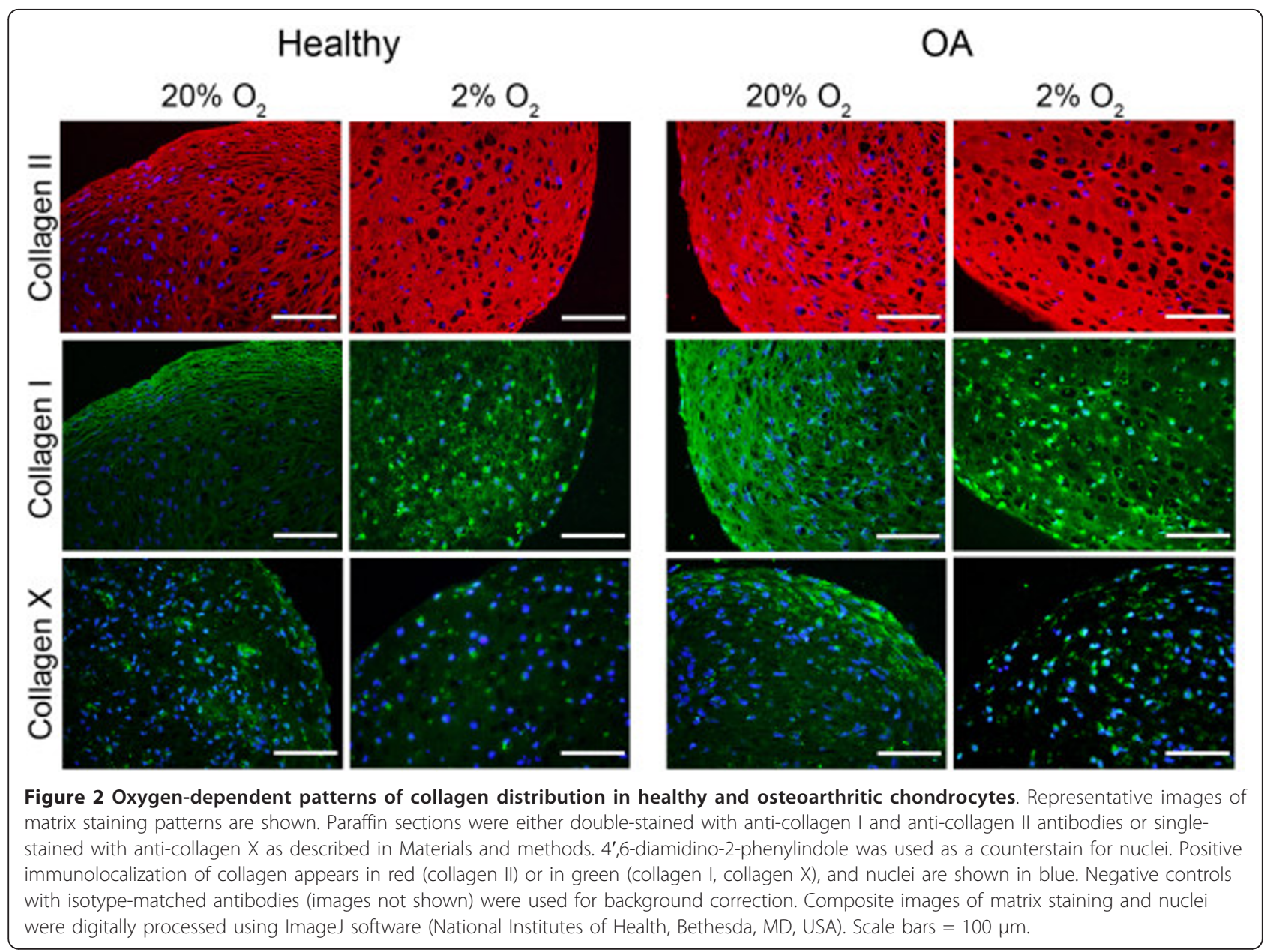

In addition to genes involved in modulating degradation, we evaluated expression of HAS2 (Figure 6A), a candidate enzyme for improving proteoglycan retention through hyaluronic acid production. HAS2 was significantly higher in hypoxic conditions in OA chondrocytes; in healthy cells, though it trended in the same direction, the difference in expression between $2 \%$ and $20 \%$ oxygen was nonsignificant $(P=0.150)$. As with ADAMTS4 and ADAMTS5 in the OA cells, HAS2 was lower in $20 \%$ oxygen in all but one of the healthy donors' chondrocyte pellets. Additionally, bivariate analysis revealed a strong and statistically significant correlation between the oxygen-dependent difference in $H A S 2$ and that of sGAG retention in both OA cells (Pearson's $r=0.909, P=$ 0.032 ) (Figure 6B) and healthy cells (Pearson's $r=0.927$, $P=0.023$ ) (Figure 6C). No significant correlation was found for either ADAMTS4 or ADAMTS5.

\section{HIF- $1 \alpha$ and HIF- $2 \alpha$ expression during chondrocyte redifferentiation}

Representative images of the patterns of HIF- $1 \alpha$ and HIF- $2 \alpha$ expression for chondrocyte pellets are shown in
Figure 7. Expression patterns of both HIF- $1 \alpha$ and HIF- $2 \alpha$ in healthy and OA chondrocytes were indistinguishable. This was consistent with mRNA data for HIF1 and EPAS1, which showed no significant differences between healthy and OA cells at day 0 or at day 14, regardless of oxygen level (data not shown). Little to no HIF-1 $\alpha$ or HIF- $2 \alpha$ was detected throughout $20 \%$ oxygen cultures. In contrast, both healthy and OA chondrocytes expressed HIF- $1 \alpha$ and HIF- $2 \alpha$ at $2 \%$ oxygen, with the strongest signals occurring early (day 2 ). When day 2 protein from hypoxic pellets was run on the same gel, we could not determine a disease-dependent difference in band intensity for either HIF- $1 \alpha$ or HIF- $2 \alpha$ (data not shown). In addition to the bands detected at the predicted molecular weights, there were other positive bands on the immunoblots. For HIF-1 $\alpha$, the additional bands were determined to be specific, with a $\lambda$-phosphatase digestion indicating that they were due to phosphorylation (data not shown). For HIF-2 $\alpha$, only the band of the predicted molecular weight had oxygen-dependent expression; the identities of the other bands could not be confirmed (Figure 7, Additional file 3 and Additional file 4). 


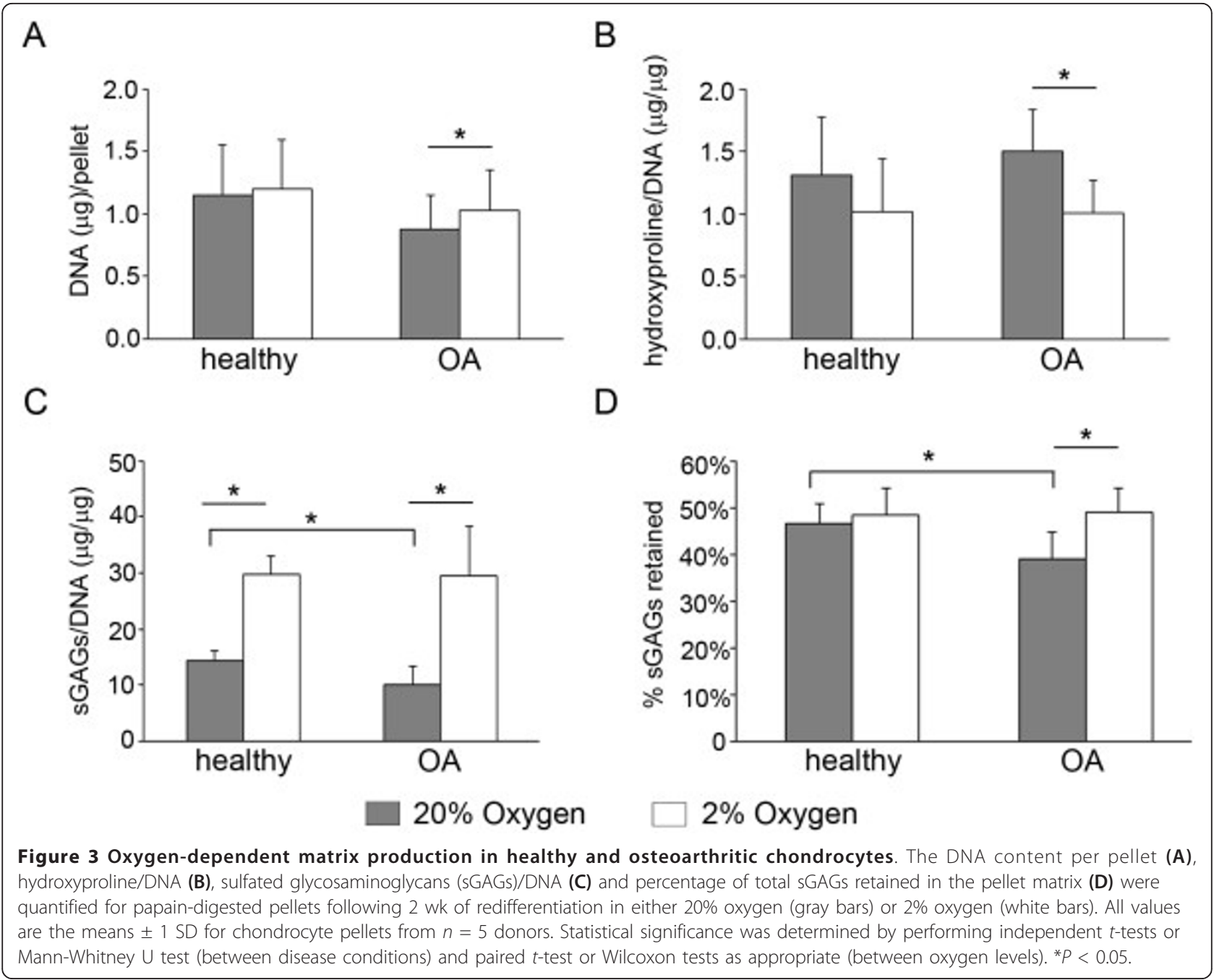

\section{Discussion}

In this study, we evaluated the oxygen-dependent response of healthy and OA chondrocytes during redifferentiation. We hypothesized that OA chondrocytes might have a different response to changes in oxygen levels compared with healthy chondrocytes, such that reduced oxygen would promote hypertrophic and degenerative changes in OA chondrocytes concomitant with different HIF expression patterns. However, our data indicate that redifferentiation was promoted, hypertrophic and OA-associated degradative genes were suppressed and HIF expression patterns were indistinguishable in both healthy and OA chondrocytes in hypoxic conditions.

Healthy and OA chondrocytes both responded with higher COL2A1 and ACAN and lower COL1A1 expression in hypoxic conditions. Hypoxic culture has previously been found to increase COL2A1 and ACAN in nondiseased chondrocytes $[8,9]$ and independently in chondrocytes from joint arthroplasties [24]. In a direct comparison, we found no differences in expression of these genes between healthy and diseased cells, regardless of oxygen tension. Additionally, though COL1A1 was higher in OA chondrocytes than in healthy chondrocytes at $20 \%$ oxygen, lower COL1A1 and less collagen I matrix staining was associated with all cells redifferentiated in hypoxia. Collagen prolyl hydroxylases require molecular oxygen, and the decreases in hydroxyproline content at $2 \%$ oxygen indicate that this level of hypoxia impairs the activity of these enzymes. It is possible that, when oxygen levels are low enough that these enzymes are impaired in chondrocytes, prolyl hydroxylation and thus secretion of collagen type II is preferentially maintained over other collagens. Consistent with this theory, the COL2A1: COL1A1 ratio was consistently elevated by hypoxia in our studies.

We also found that hypoxia increased proteoglycan accumulation by both healthy and OA cells. Excess oxygen may be more detrimental to cells from OA joints, 


\section{$20 \%$ Oxygen $\square 2 \%$ Oxygen}

A

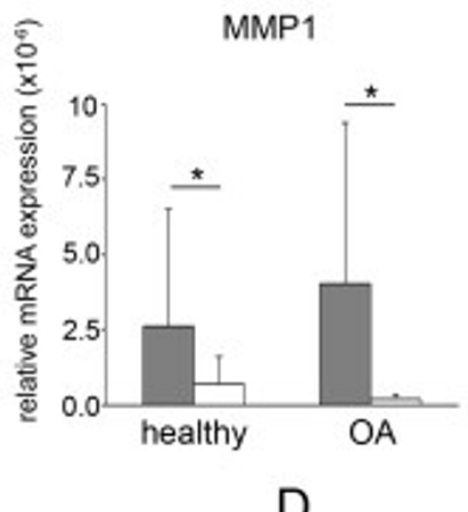

D

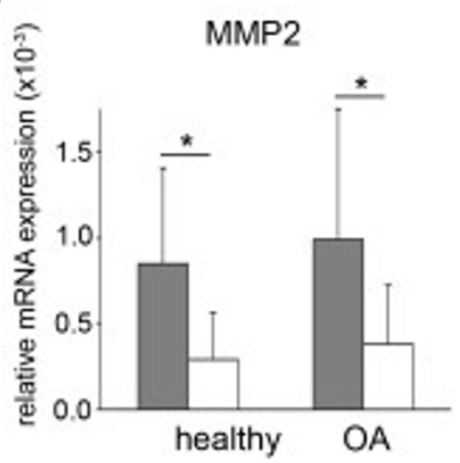

$\mathrm{F}$

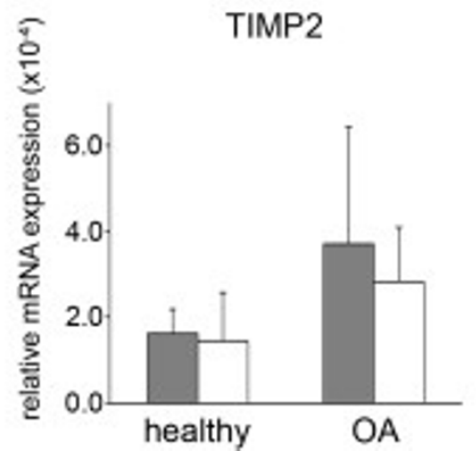

B

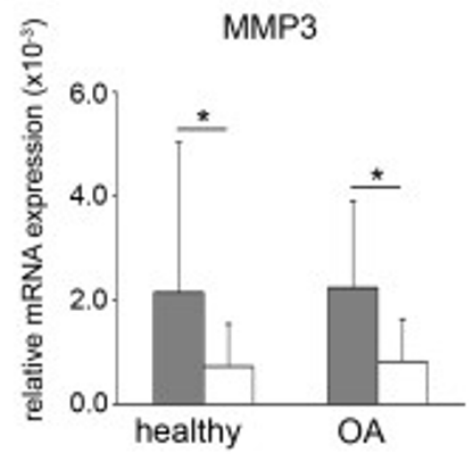

C

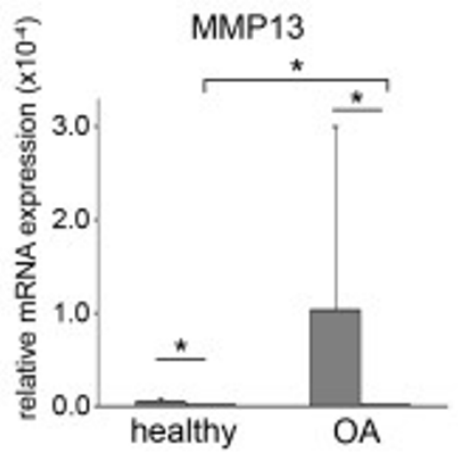

E

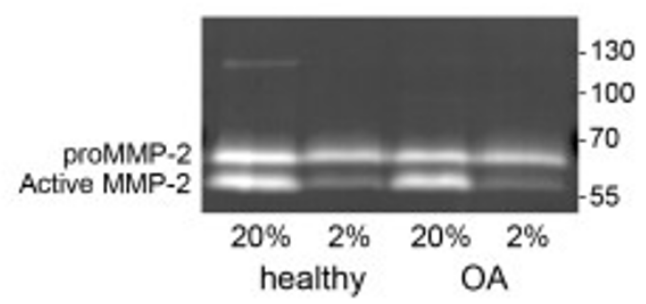

G

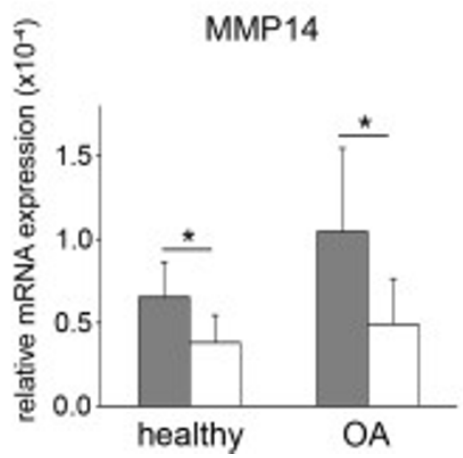

Figure 4 Oxygen-dependent expression of matrix metalloproteinases in healthy and osteoarthritic chondrocytes. (A) through (D) The relative gene expression levels of MMP1, MMP3, MMP13 and MMP2 in pellet cultures following 2 wk of redifferentiation in either 20\% oxygen (gray bars) or $2 \%$ oxygen (white bars) were analyzed by real-time quantitative polymerase chain reaction. (E) Gelatin zymography of supernatants pooled from days 9 to 14 of pellet cultures indicates levels of secreted pro-MMP2 and active MMP2 in healthy and osteoarthritic (OA) cells at 20\% and 2\% oxygen. (F) and (G) The relative gene expression levels of TIMP2 and MMP14 in pellet cultures following 2 wk of redifferentiation in either $20 \%$ oxygen (gray bars) or $2 \%$ oxygen (white bars) were analyzed by real-time quantitative polymerase chain reaction. All expression values are the mean mRNA levels normalized to $18 \mathrm{~S}$ ribosomal RNA for chondrocyte pellets from $n=5$ donors. Error bars represent 1 SD. Statistical significance was determined by performing independent $t$-tests (between disease conditions) and paired $t$-tests (between oxygen levels). ${ }^{*} P<0.05$.

however, because OA cell pellets had significantly less sGAG/DNA than those of healthy cells maintained at high oxygen. Whether this is due to a greater oxygen sensitivity that results in impaired synthesis, more degradation, disturbed matrix assembly or some combination of these processes remains to be determined. The percentage of sGAGs retained within the matrix was also significantly reduced in OA chondrocytes at $20 \%$ oxygen. This may be 

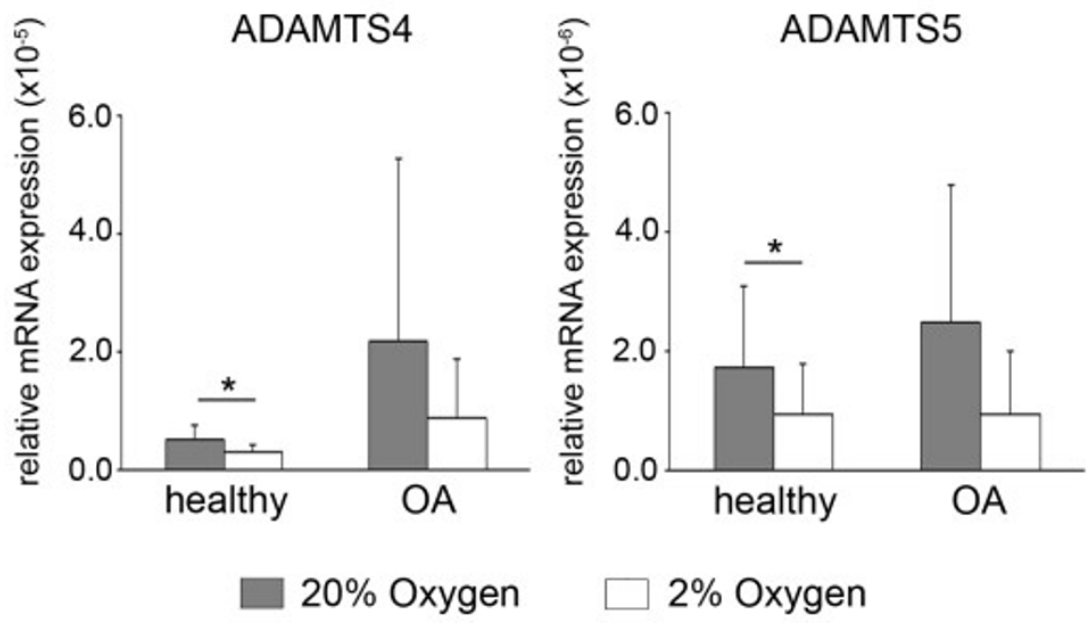

Figure 5 Oxygen-dependent expression of aggrecanases in healthy and osteoarthritic chondrocytes. The relative gene expression levels of ADAMTS4 (A) and ADAMTS5 (B) in pellet cultures following 2 wk of redifferentiation in either $20 \%$ oxygen (gray bars) or 2\% oxygen (white bars) were analyzed by real-time quantitative polymerase chain reaction. All values are the mean mRNA levels normalized to $18 \mathrm{~S}$ ribosomal RNA for chondrocyte pellets from $n=5$ donors. Error bars represent 1 SD. Statistical significance was determined by performing independent $t$-tests (between disease conditions) and paired $t$-tests (between oxygen levels). ${ }^{*} P<0.05$.

indicative of poorer proteoglycan incorporation into the matrix or of increased degradation. With regard to the former possibility, we assessed expression of HAS2, the predominant form of the HAS enzyme in chondrocytes $[25,26]$. Previously, studies using RNA interference against $H A S 2$ showed that this did not affect $A C A N$ expression or proteoglycan synthesis, but that it did decrease the amount of proteoglycans retained within the matrix [25]. HAS2 expression and hyaluronic acid synthesis have also been shown to be oxygen-dependent in bovine chondrocytes [27]. We found that $20 \%$ oxygen resulted in lower mean HAS2 expression in both healthy and OA chondrocytes. Furthermore, there was a strong correlation between the oxygen-dependent difference in HAS2 and that of sGAG retention in both healthy and OA cells. Reduced hyaluronic acid synthesis could allow for more passive loss of

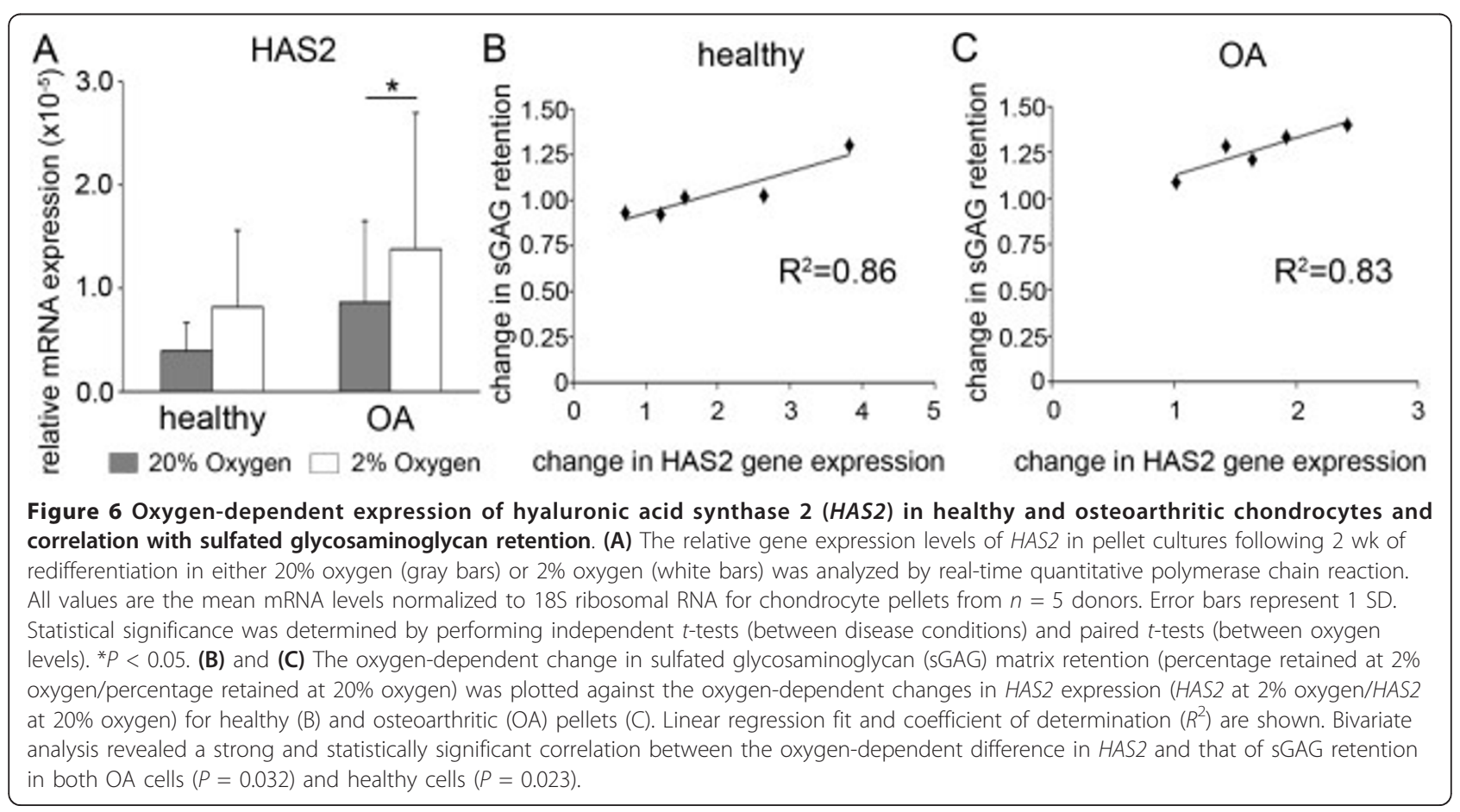




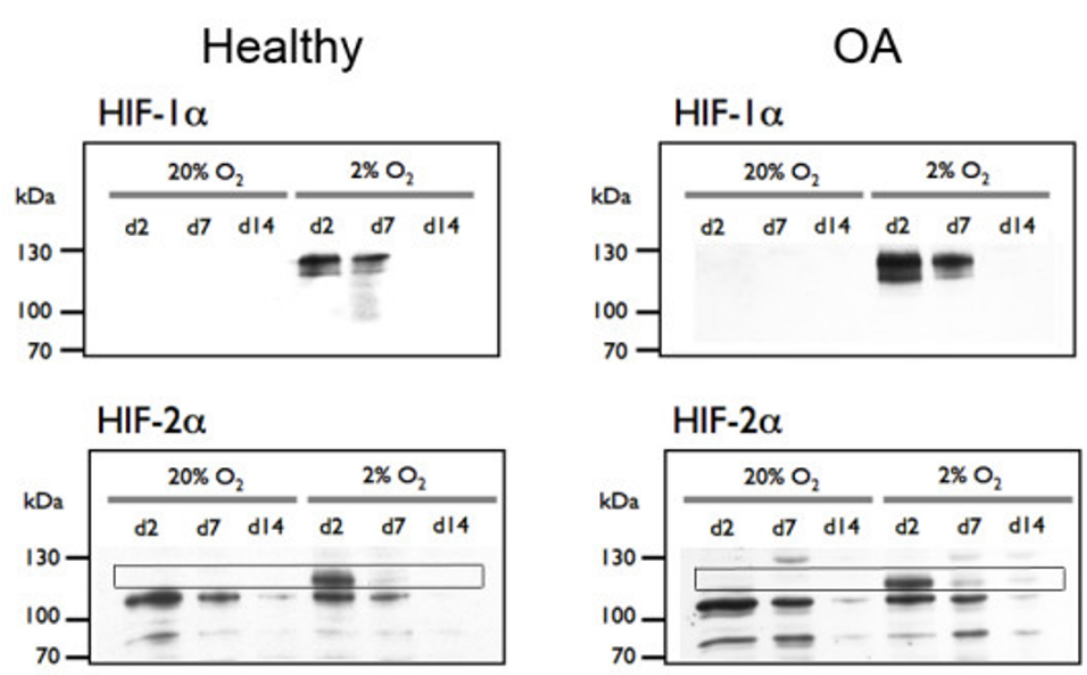

Figure 7 Hypoxia-inducible factors $1 \alpha$ and $2 \alpha$ protein expression during redifferentiation of healthy and osteoarthritic chondrocytes. Representative images of banding patterns are shown. Normalization of nuclear protein loading was achieved by first running equal volumes of each sample and probing for histone H3. For hypoxia-inducible factor (HIF) blots, the volume loaded was adjusted to give equivalent nuclear loading based on each histone $\mathrm{H} 3$ band's signal relative to the signal from the lowest-intensity band. The location of the only oxygendependent band on HIF-2 $\alpha$ blots is in the boxed area, and the blot is cropped to show only the bands in the molecular weight range where the HIF-2 $\alpha$ product is predicted (115 kDa). Unmodified HIF-2 $\alpha$ blots for healthy and OA chondrocytes are shown in Additional files 3 and 4 , respectively. The consistency of the nonspecific band near $35 \mathrm{kDa}$ (lowest band) was used as a loading control after histone $\mathrm{H} 3 \mathrm{normalization}$.

proteoglycans; however, hyaluronic acid has also been reported to modulate expression and/or activity of ADAMTS4 [28] and MMP13 [29] in chondrocytes. Determining specific mechanisms by which HAS2 modulation by oxygen affects chondrocytes and their matrix requires further investigation.

The potent anabolic effects of hypoxia on chondrocytes are well-documented, but less is known about its regulation of catabolism. In the two studies implicating HIF- $2 \alpha$ in hypertrophy and $\mathrm{OA}, M M P 3, M M P 9 / M m p 9$ and MMP13 were reported to be upregulated by HIF- $2 \alpha$ $[12,13]$. One of the studies also reported $M M P 1$ and Adamts4, but not Adamts5, to be promoted by HIF- $2 \alpha$ [13], but the other found no association for either Adamts4 or Adamts5. In contrast, hypoxia reportedly decreases MMP1 and MMP13 expression and collagenolytic activity in healthy human chondrocytes and decreases ADAMTS5 and aggrecanase activity in healthy human cartilage explants $[10,11]$. We found that $M M P 1$ and $M M P 13$ were lower in hypoxia not only in healthy chondrocytes but also in OA chondrocytes. Additionally, MMP2 and $M M P 3$ were lower in both healthy and OA chondrocytes in hypoxic culture, and less of the active form of MMP-2 was generated, concomitant with lower expression of $M M P 14$. As reported by Ströbel et al., we detected no oxygen-dependent changes in $M M P 9$ expression, because this gene was at the limit for detection in both healthy and OA cells [11]. ADAMTS4 and ADAMTS5 were also lower in both healthy and OA chondrocytes in hypoxia, but the differences were significant only in healthy cells. Although hypoxia was associated with transcriptional downregulation of these enzymes, we did not address the potential for oxygen-dependent activity. Like MMPs, the ADAMTS proteases are synthesized as inactive zymogens and assessing whether and to what extent their activation is oxygen-dependent is an essential next step in understanding the role of oxygen in cartilage degeneration. Recent findings by Thoms et al. indicate that hypoxic culture does indeed suppress loss of aggrecanase-generated aggrecan fragments from healthy cartilage explants and that TIMP3 may play a role in the oxygen-dependent inhibition [10].

We found that COL1OA1 was dramatically lower at $2 \%$ oxygen compared with $20 \%$ oxygen in both healthy and OA chondrocytes. This finding contrasts with that of Schrobback et al., who reported that COL10A1 was not oxygen-dependent in chondrocytes from patients undergoing arthroplasty [30]. However, they used 5\% oxygen, and, though it was reported as nonsignificant, COL10A1 was on average lower at $5 \%$ oxygen than at $20 \%$ oxygen. Because our data suggest that OA chondrocytes may be particularly sensitive to oxygen, the different oxygen levels could explain the discrepancies. Compared to standard culture conditions, human neuroblastoma cells grown at $5 \%$ oxygen were reported to have elevated levels of HIF- $2 \alpha$ only, whereas those grown at $1 \%$ oxygen had increased levels of both HIF-1 $\alpha$ and HIF-2 $\alpha$ [31]. Although the relative contributions of the HIFs to collagen type expression remain unknown, we observed 
increased levels of both HIF- $1 \alpha$ and HIF- $2 \alpha$ at $2 \%$ oxygen. If expression patterns of the HIFs vary in chondrocytes at higher oxygen levels, this could alter collagen expression profiles.

Our finding that hypoxia decreases COL10A1 also seems to be at odds with results reported by Saito et al. indicating that HIF- $2 \alpha$ was a potent transactivator of COL10A1, at least when paired with the $\beta$-subunit of ARNTL [12]. This discrepancy is not inexplicable, because it is known that HIFs are posttranslationally modified in a number of ways that may be dependent on culture conditions, including oxygen tension, and we performed our studies in hypoxic conditions, whereas Saito et al. did theirs at $20 \%$ oxygen. For example, both HIF$1 \alpha[32,33]$ and HIF-2 $\alpha$ [34] have been reported to be phosphorylated in hypoxia. Although we cannot confirm the presence of HIF- $2 \alpha$ modifications, phosphorylation of HIF-1 $\alpha$ appears to be relevant in chondrocytes. The overexpression studies conducted in standard oxygen conditions could alter such regulatory mechanisms of HIF signaling and contribute to reported differences. With the reports of HIF-2 $\alpha$ 's involvement in promoting hypertrophic genes came suggestions that targeting HIF$2 \alpha$ could be an effective treatment for OA [17]. As was also noted, targeting the relevant dimerization partners involved in promoting these genes may be a more suitable strategy. Our results support this alternate view, because the absence of HIF expression in human chondrocytes is associated with increased markers of hypertrophy and degeneration and the banding patterns of the HIF $\alpha$-subunits were identical, regardless of the cells' origin.

Although there were no obvious differences in the response of HIF- $1 \alpha$ and HIF- $2 \alpha$ expression between healthy and OA cells, because more of the measured outcomes were affected by high oxygen in OA chondrocytes, we cannot rule out that there are differences in oxygendependent signaling in OA chondrocytes compared with healthy chondrocytes. With higher oxygen exposure, OA chondrocytes have significantly higher COL1A1 and COL10A1 mRNA expression compared with healthy chondrocytes and exhibit loss of proteoglycans from the matrix in addition to having lower cell numbers at the end of the culture period compared with their hypoxic counterparts. Although there were differences between redifferentiated healthy and OA chondrocytes that were evident or more pronounced only under higher oxygen tension, only the expression levels of COL1OA1 and $M M P 13$ were significantly different between healthy and OA cells under hypoxic conditions. There may be HIF signaling-related mechanisms that are responsible for the disease state-dependent differences we see in certain parameters of redifferentiation. The $\alpha$-subunits are generally assumed to be the rate-limiting factors because they are specific to hypoxic targets, unlike the promiscuous $\beta$-subunits. However, we cannot rule out that the presence and relative ratios of the $\beta$-subunits and control of dimerization could affect HIF activity in ways that would not be detected by Western blot analysis for HIF $\alpha$-subunits. In addition, the asparginyl hydroxylase factor inhibiting HIF, which blocks coactivator recruitment, may be differentially regulated. Furthermore, epigenetic phenomena have been shown to be involved in the regulation of HIF target gene expression in chondrocytes. Hashimoto et al. demonstrated that methylation of CpG sites in the hypoxia-responsive element in the proximal promoter region of MMP13 inhibited HIF-2 $\alpha$-dependent transactivation [35]. This is an intriguing line of inquiry and may provide a valuable key to understanding the patterns of gene expression we describe and possibly even a mechanism for the donor-to-donor variation observed with primary cells.

\section{Conclusions}

Hypoxic culture lessens the differences between OA and healthy chondrocytes, and the effect of elevated oxygen leads to changes in both healthy and OA chondrocytes that are generally associated with degenerative cartilage. This implies that a loss of HIF signaling could be involved in promoting changes associated with cartilage degradation. However, these studies were done in the absence of external stress signals, such as inflammatory factors, which could modulate HIF signaling and alter the downstream effects. That the detrimental effects of high oxygen are greater in the OA chondrocytes indicates that diseased cells may be less equipped to mitigate the stress of impaired HIF signaling and that this difference is maintained through in vitro expansion.

\section{Additional material}

Additional file 1: Macroscopic and toluidine blue-stained sections of
healthy and osteoarthritic cartilage specimens. Representative
cartilage specimen images of healthy (A) and (C) and OA (B) and (D)
are shown.
Additional file 2: Ratio of COL2A1 to COL1A1 expression in healthy
and osteoarthritic chondrocytes. The ratios of the relative gene
expression levels of COL $2 A 1$ and COL $1 A 1$ in pellet cultures following 2 wk
of redifferentiation in either $20 \%$ oxygen (gray bars) or $2 \%$ oxygen (white
bars) were calculated. All values are the mean ratios from $n=5$ donors.
Error bars represent 1 SD. Statistical significance was determined by
performing independent $t$-tests (between disease conditions) and paired
-tests (between oxygen levels). *P $<0.05$.
Additional file 3 : Full hypoxia-inducible factor $2 \alpha$ blot for healthy
chondrocytes shown in Figure 7 . Unmodified version of healthy
chondrocyte HIF-2 $\alpha$ blot shown in Figure 7 .
Additional file 4 : Full hypoxia-inducible factor $2 \alpha$ blot for
osteoarthritic chondrocytes shown in Figure 7 . Unmodified version of
OA chondrocyte HIF-2 $\alpha$ blot shown in Figure 7 .




\section{Abbreviations}

ACAN: human aggrecan gene; Adamts4/ADAMTS4: mouse/human aggrecanase 1 gene; Adamts5/ADAMTS5: mouse/human aggrecanase 2 gene; BSA: bovine serum albumin; COL1A1: human collagen type l, a1 gene; COL2A1: human collagen type II, a1 gene; COL10A1: human collagen type $X$, a1 gene; DMEM: Dulbecco's modified Eagle's medium; DMMB: 1,9dimethymethylene blue; Epas1/EPAS1: mouse/human endothelial Per-ArntSim domain-containing protein 1 (HIF-2a) gene; HAS2: human hyaluronic acid synthase 2 gene; HIF: hypoxia-inducible factor; MMP: matrix metalloproteinase; OA: osteoarthritis; PAS: Per-Arnt-Sim domain; P/S penicillin-streptomycin; sGAG: sulfated glycosaminoglycan; TIMP2: human tissue inhibitor of metalloproteinases 2 gene.

\section{Competing interests}

The authors declare that they have no competing interests.

\section{Authors' contributions}

BDM participated in study conception and design and data interpretation, coordinated the experiments, acquired data (biochemistry, GPCR and immunohistochemistry), performed the statistical analyses and drafted the manuscript. HC participated in study conception and design, data acquisition and interpretation (immunohistochemistry, zymography and Western blotting) and helped to draft and critically revise the manuscript. BJ participated in study conception and design and data interpretation and critically revised the manuscript. All authors read and approved the final manuscript.

\section{Acknowledgments}

The authors thank Drs Dennis Crawford and Tom Huff (OHSU Department of Orthopaedics) for providing human healthy and OA cartilage samples. We are grateful to Dr Paul Holden (OHSU Department of Orthopaedics) for guidance in the technical aspects of protein extraction, HIF immunoblotting and zymography and to Prof Jung Yoo (OHSU Department of Orthopaedics) for consultation on appropriate statistical analysis. This research was supported in part by grants from the Collins Medical Trust and the Arthritis Foundation.

Received: 8 March 2013 Accepted: 21 August 2013

Published: 21 August 2013

\section{References}

1. Schiltz JR, Mayne R, Holtzer H: The synthesis of collagen and glycosaminoglycans by dedifferentiated chondroblasts in culture. Differentiation 1973, 1:97-108.

2. Benya PD, Shaffer JD: Dedifferentiated chondrocytes reexpress the differentiated collagen phenotype when cultured in agarose gels. Cell 1982, 30:215-224.

3. Dehne T, Karlsson C, Ringe J, Sittinger M, Lindahl A: Chondrogenic differentiation potential of osteoarthritic chondrocytes and their possible use in matrix-associated autologous chondrocyte transplantation. Arthritis Res Ther 2009, 11:R133.

4. Tallheden T, Bengtsson C, Brantsing C, Sjögren-Jansson E, Carlsson L, Peterson L, Brittberg M, Lindahl A: Proliferation and differentiation potential of chondrocytes from osteoarthritic patients. Arthritis Res Ther 2005, 7:R560-R568.

5. Araldi E, Khatri R, Giaccia AJ, Simon MC, Schipani E: Lack of HIF-2a in limb bud mesenchyme causes a modest and transient delay of endochondral bone development. Nat Med 2011, 17:25-29.

6. Murphy CL: HIF-2a: a mediator of osteoarthritis? Cell Res 2010, 20:977-979.

7. Nakajima M, Shi D, Dai J, Tsezou A, Zheng M, Norman PE, Chou CH, Lee MT, Hwang JY, Kim DH, Takahashi A, Ikegawa S, Jiang Q: A large-scale replication study for the association of rs 17039192 in HIF-2a with knee osteoarthritis. J Orthop Res 2012, 30:1244-1248.

8. Lafont JE, Talma S, Murphy CL: Hypoxia-inducible factor $2 a$ is essential for hypoxic induction of the human articular chondrocyte phenotype. Arthritis Rheum 2007, 56:3297-3306.

9. Murphy CL, Polak JM: Control of human articular chondrocyte differentiation by reduced oxygen tension. J Cell Physiol 2004, 199:451-459.

10. Thoms BL, Dudek KA, Lafont JE, Murphy CL: Hypoxia promotes the production and inhibits the destruction of human articular cartilage. Arthritis Rheum 2013, 65:1302-1312.
11. Ströbel S, Loparic M, Wendt D, Schenk AD, Candrian C, Lindberg RL, Moldovan F, Barbero A, Martin I: Anabolic and catabolic responses of human articular chondrocytes to varying oxygen percentages. Arthritis Res Ther 2010, 12:R34.

12. Saito T, Fukai A, Mabuchi A, Ikeda T, Yano F, Ohba S, Nishida N, Akune T, Yoshimura N, Nakagawa T, Nakamura K, Tokunaga K, Chung UI, Kawaguchi H: Transcriptional regulation of endochondral ossification by HIF-2a during skeletal growth and osteoarthritis development. Nat Med 2010, 16:678-686.

13. Yang S, Kim J, Ryu JH, Oh H, Chun CH, Kim BJ, Min BH, Chun JS: Hypoxiainducible factor-2 $\alpha$ is a catabolic regulator of osteoarthritic cartilage destruction. Nat Med 2010, 16:687-693.

14. Bohensky J, Terkhorn SP, Freeman TA, Adams CS, Garcia JA, Shapiro IM, Srinivas $\mathrm{V}$ : Regulation of autophagy in human and murine cartilage: hypoxia-inducible factor 2 suppresses chondrocyte autophagy. Arthritis Rheum 2009, 60:1406-1415.

15. Pfander D, Cramer T, Swoboda B: Hypoxia and HIF-1a in osteoarthritis. Int Orthop 2005, 29:6-9.

16. Yudoh K, Nakamura H, Masuko-Hongo K, Kato T, Nishioka K: Catabolic stress induces expression of hypoxia-inducible factor (HIF)-1a in articular chondrocytes: involvement of HIF-1a in the pathogenesis of osteoarthritis. Arthritis Res Ther 2005, 7:R904-R914.

17. Husa M, Liu-Bryan R, Terkeltaub R: Shifting HIFs in osteoarthritis. Nat Med 2010, 16:641-644.

18. Ruiz-Romero C, Calamia V, Rocha B, Mateos J, Fernández-Puente P, Blanco FJ: Hypoxia conditions differentially modulate human normal and osteoarthritic chondrocyte proteomes. J Proteome Res 2010, 9:3035-3045.

19. Clérigues $\vee$, Murphy $C L$, Guillén Ml, Alcaraz MJ: Haem oxygenase-1 induction reverses the actions of interleukin-1 $\beta$ on hypoxia-inducible transcription factors and human chondrocyte metabolism in hypoxia. Clin Sci (Lond) 2013, 125:99-108.

20. Jakob M, Démarteau O, Schäfer D, Hintermann B, Dick W, Heberer M, Martin I: Specific growth factors during the expansion and redifferentiation of adult human articular chondrocytes enhance chondrogenesis and cartilaginous tissue formation in vitro. J Cell Biochem 2001, 81:368-377.

21. Ball ST, Amiel D, Williams SK, Tontz W, Chen AC, Sah RL, Bugbee WD: The effects of storage on fresh human osteochondral allografts. Clin Orthop Relat Res 2004, 418:246-252.

22. Angele P, Yoo JU, Smith C, Mansour J, Jepsen KJ, Nerlich M, Johnstone B: Cyclic hydrostatic pressure enhances the chondrogenic phenotype of human mesenchymal progenitor cells differentiated in vitro. J Orthop Res 2003, 21:451-457.

23. Holden $\mathrm{P}$, Horton WA: Crude subcellular fractionation of cultured mammalian cell lines. BMC Res Notes 2009, 2:243.

24. Duval E, Leclerca S, Elissalde JM, Demoor M, Galéra P, Boumédiene K Hypoxia-inducible factor $1 a$ inhibits the fibroblast-like markers type I and type III collagen during hypoxia-induced chondrocyte redifferentiation: hypoxia not only induces type II collagen and aggrecan, but it also inhibits type I and type III collagen in the hypoxiainducible factor 1a-dependent redifferentiation of chondrocytes. Arthritis Rheum 2009, 60:3038-3048.

25. Nishida Y, Knudson CB, Nietfeld JJ, Margulis A, Knudson W: Antisense inhibition of hyaluronan synthase- 2 in human articular chondrocytes inhibits proteoglycan retention and matrix assembly. J Biol Chem 1999, 274:21893-21899.

26. Recklies AD, White $C$, Melching $L$, Roughley PJ: Differential regulation and expression of hyaluronan synthases in human articular chondrocytes, synovial cells and osteosarcoma cells. Biochem J 2001, 354:17-24.

27. Hashimoto K, Fukuda K, Yamazaki K, Yamamoto N, Matsushita T, Hayakawa S, Munakata H, Hamanishi C: Hypoxia-induced hyaluronan synthesis by articular chondrocytes: the role of nitric oxide. Inflamm Res 2006, 55:72-77.

28. Yatabe T, Mochizuki S, Takizawa M, Chijiiwa M, Okada A, Kimura T, Fujita Y, Matsumoto $H$, Toyama Y, Okada Y: Hyaluronan inhibits expression of ADAMTS4 (aggrecanase-1) in human osteoarthritic chondrocytes. Ann Rheum Dis 2009, 68:1051-1058.

29. Julovi SM, Ito H, Nishitani K, Jackson CJ, Nakamura T: Hyaluronan inhibits matrix metalloproteinase-13 in human arthritic chondrocytes via CD44 and P38. J Orthop Res 2011, 29:258-264.

30. Schrobback K, Malda J, Crawford RW, Upton Z, Leavesley DI, Klein TJ: Effects of oxygen on zonal marker expression in human articular chondrocytes. Tissue Eng Part A 2012, 18:920-933. 
31. Holmquist L, Jögi A, Påhlman S: Phenotypic persistence after reoxygenation of hypoxic neuroblastoma cells. Int I Cancer 2005, 116:218-225.

32. Richard DE, Berra E, Gothié E, Roux D, Pouysségur J: p42/p44 mitogenactivated protein kinases phosphorylate hypoxia-inducible factor 1 a (HIF-1a) and enhance the transcriptional activity of HIF-1.J Biol Chem 1999, 274:32631-32637.

33. Suzuki H, Tomida A, Tsuruo T: Dephosphorylated hypoxia-inducible factor $1 \mathrm{a}$ as a mediator of p53-dependent apoptosis during hypoxia. Oncogene 2001, 20:5779-5788.

34. Conrad PW, Freeman TL, Beitner-Johnson D, Millhorn DE: EPAS1 transactivation during hypoxia requires p42/p44 MAPK. J Biol Chem 1999, 274:33709-33713.

35. Hashimoto $\mathrm{K}$, Otero M, Imagawa K, de Andrés MC, Coico JM, Roach HI, Oreffo RO, Marcu KB, Goldring MB: Regulated transcription of human matrix metalloproteinase 13 (MMP13) and interleukin-1 1 (IL1 $\beta)$ genes in chondrocytes depends on methylation of specific proximal promoter CpG sites. J Biol Chem 2013, 288:10061-10072.

doi:10.1186/ar4272

Cite this article as: Markway et al:: Hypoxia promotes redifferentiation and suppresses markers of hypertrophy and degeneration in both healthy and osteoarthritic chondrocytes. Arthritis Research \& Therapy 2013 15:R92.

\section{Submit your next manuscript to BioMed Central and take full advantage of:}

- Convenient online submission

- Thorough peer review

- No space constraints or color figure charges

- Immediate publication on acceptance

- Inclusion in PubMed, CAS, Scopus and Google Scholar

- Research which is freely available for redistribution

Submit your manuscript at www.biomedcentral.com/submit 